\title{
THE DESIGN OF EMBEDDED VIDEO SUPERVISION SYSTEM OF VEGETABLE SHED
}

\author{
Jun Sun $^{1,2, *}$, Mingxing Liang ${ }^{2}$, Weijun Chen ${ }^{2}$, Bin Zhang ${ }^{3}$ \\ ${ }^{1}$ Key Laboratory of Modern Agricultural Equipment and Technology, inistry of Education \& \\ Jiangsu Province, Jiangsu University, Zhenjiang, Jiangsu Province, China 212013 \\ ${ }^{2}$ School of Electrical and Information Engineering, Jiangsu university, Zhenjiang ,Jiangsu \\ Province, China 212013 \\ ${ }^{3}$ ChangZhou Institute of Technology, Changzhou,Jiangsu Province, China 213002 \\ * Corresponding author,Address:School of Electrical and Information Engineering, Jiangsu \\ University, Zhenjiang 212013,Jiangsu Province,China, Tel:(0)13775544650, Fax:0511887 \\ 80311,Email:sun2000jun@ujs.edu.cn
}

Abstract: In order to reinforce the measure of vegetable shed's safety, the S3C44B0X is taken as the main processor chip. The embedded hardware platform is built with a few outer-ring chips, and the network server is structured under the Linux embedded environment, and MPEG4 compression and real time transmission are carried on. The experiment indicates that the video monitoring system can guarantee good effect, which can be applied to the safety of vegetable sheds.

Keywords: embedded system, safety monitoring, MPEG4

\section{INTRODUCTION}

Along with the function of the vegetable sheds construction being perfected day by day, it turns to be an important topic for the technical personnel to strengthen the safe management of the vegetable sheds. The former small scaled vegetable sheds sends a person on duty by turn inside the shed. This kind of way can attain the purpose of defending, but it also needs a large amount of labor and wastes lots of human resources. At present, some vegetable sheds adopt surveillance nearby the sheds. This method carries out the supervision of all important equipments of the shed, but it is

\footnotetext{
Please use the following format when citing this chapter:
}

Sun, J., Liang, M., Chen, W. and Zhang, B., 2009, in IFIP International Federation for Information Processing, Volume 294, Computer and Computing Technologies in Agriculture II, Volume 2, eds. D. Li, Z. Chunjiang, (Boston: Springer), pp. 1329-1335. 
limited to only a short distance(Kallesoe,2004;Behzad,2004;Zhang,2006). With the fast development of the communication technique of the calculator network and the compress technique in multi-media graphs, a new way for the video supervision in a long range can be provided, in which governor can control the actual condition in the shed much more easily.

Traditional supervision system uses a video frequency collection card to set up a network video frequency in the PC. However, the performance of real-time and opening is not good, price is high, and the extent of integration and the stability of system is bad. At present, in the digital monitoring system, embedded technology and MPEG-4 compress coding technology are doubly subjected to concern. Development of embedded MPEG-4 digital video surveillance system has higher technology and good market prospect. This literature is mainly about the research of the supervision and regulation of the vegetable sheds based on embedded system and network technology.

\section{SYSTEM MODEL}

We set cameras in key positions both in and out of the vegetable sheds house and use analog shoots to carry on the real-time analog graphs of the shed scene. Because of the special environment of the vegetable sheds, the lighting in night and even the rainy and windy weather should be considered. So in order to collect the video frequency of high quality, generally we choose low shine shoots. The numerical video frequency signal conversion mold piece can change the analog graphs into digital pictures, while the compress mold can reduce the saving space of video frequency pictures. With a network video frequency server built up, users only need to put the IP address of the server into the IE browser address column to browse the picture.

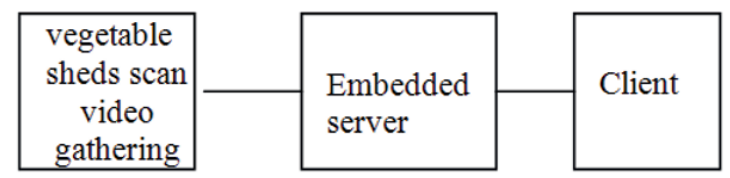

Fig. 1 The whole frame image of video monitoring system

\section{HARDWARE DESIGN OF EMBEDDED SERVER}

The hardware structure includes the video frequency A/D chip SAA7111, core processors ARM7 embedded chip S3C44BOX, MPEG compress chip IME6400, fast Ethernet physical layer chip RTL8019AS. The system hardware frame shows in figure 2. 


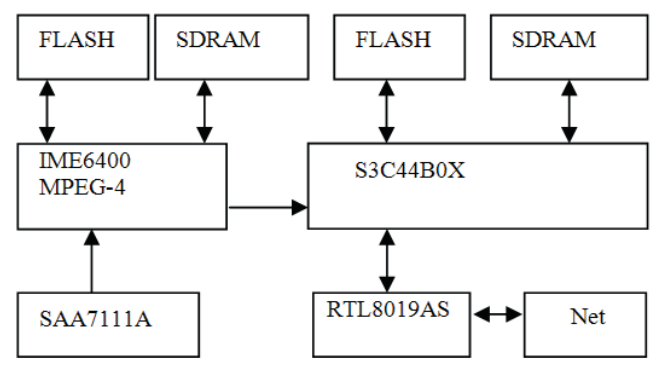

Fig.2: The hardware frame image of embedded server

SAA7111A is a kind of enhanced video input processor chip made by PHILIPS, which gathers A/D and decoding at the whole body. Both of the PAL and the NTSC TV standard are fit. The SAA7111A contains I2C interface internal through which work conditions can be set. Signals of SAA7111A such as VREF, HREF, RESO and LLC2 are all lead directly from the pins and have good reliability. Though A/D converter, the video frequency collection mold piece change the original analog video signals into digital signals. In order to deliver the video frequency signal on the net which is limited by its bandwidth, the numerical signal needs chip IME6400 to carry on MPEG4 compressions and then be sent to embedded processor S3C44BOX. In the end, the compression data is sent to Internet through the interface RTL8019AS and RJ45. Users can access the video data which is sent by the network interface to the network by WEB server( Xue., 2006) .

The IME6400 delivers flow for network application or program flow for saving application. The IME6400 is a kind of ideal chip made by INTIME which carries out MPEG-4 compression. Compared with MPEG-1 and MPEG-2, MPEG-4 standard has a higher compression ratio, saving storage space and better image quality. Specially, under the low bandwidth, it can adjust the bit rate of the compressed data from $128 \mathrm{Kbps}$ to $6 \mathrm{Mbps}$, while keeping the quality of the pictures to adapt to various demands of users(Jia.,2005) .

RTL8019AS is a kind of highly integrated Ethernet chip, compatible with NE2000 and supporting IEEE802.3 standard. It can install into 8 or 16 lines with total data. With 16KB SRAM memory inside, the chip can be used as a receiving cache for sending data, supporting full-duplex mode. There are three kinds of the interface modes: jumper mode, non-jumper mode and plug-and-play mode, with the automatic examination equipments to check whether the plug-and-play jumper has been connected or not.

The FLASH chooses the NAND type K9F1208 of 32 Mbytes to deposit the boot codes, the kernel codes and root file systems. In the management of FLASH, root document system adopts the latest system called YAFFS which can be both read and written. We can record the users connection logs into the root file system so that it's easy for the server to call the logs. 
The RAM adopts two slices of HY57V561620, total 64 Mbytes of SDARM, facilitating the smooth running of Linux, as well as the network applications.

\section{DESIGN OF NETWORK VIDEO SERVER}

The extensive application of WEB technique on the Internet causes the popularity of B/S mode, which can be also called "thin customer's machine". With the main application procedures existing at the servers, users only demand the browser environment, then it is easy to download application procedure from the severs to accomplish the match tasks according to the needing.

The embedded network video frequency sever adopts embedded integrated structure(He.,2003; Zhang.,2003). With the hardware platform for real-time processing, both of the video frequency compression and network function can be concentrated to a small individual. After collection, compression and compounding, the video frequency signals then turn to the IP packet, and with suitable network protocol, the video compressed data stream can carry on real-time transmission. Users only need a browser to watch instead of installing any hardware equipments.

The software of the network video frequency server contains 5 main modules: system initialization, acquisition and compression, as well as the packing and transmission of the data, responding to users' requests and management of users.

System initialization module is mainly responsible for reading parameters which are important for the enactment of the server port and the network bandwidth, as well as the picture manifestation. Besides, it can also start the other mold pieces of the sever; build a set of connections with the HTTP service provided according to the port parameters; build up a conjunction, respond to the WEB interview of customers' machine and handle the claim from users.

In the module of the acquisition and compression of the video frequency data, the main mission is to collect and digitize the video analog signals. As well as, making the full use of the mature compression technique to realize high compression of the data with satisfying the technique index is also very important. This system adopts the MPEG-4 chip to carry on the compression and coding of the original data, then put into the buffer area. It turns easy to carry on the compression and coding of the video with the use of the member function. After the setting parameters are delivered into a type, the member function can work such as showing in figure 3. 


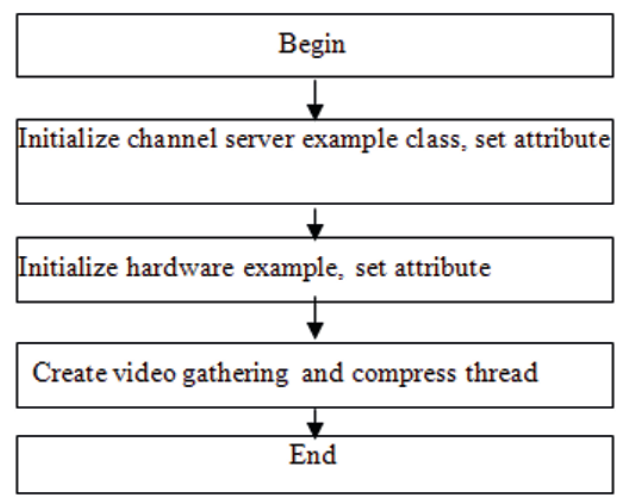

Fig.3: The flow image of video's capturing and compression

The module of packing and transmission of the data compress the data collected into the MPEG-4 format, and then carry on packing according to the RTP protocol. Finally, it's sent out to the particular port of the users who ask for help through the network. The member function of type RTPSeesion reads video frequency data from the buffer area, and then begins to pack. Before the packing, the data needs to be incised into some segments, and then plus the RTP protocol as a heading file to each part. The sending course of the video frequency flow shows in figure 4.

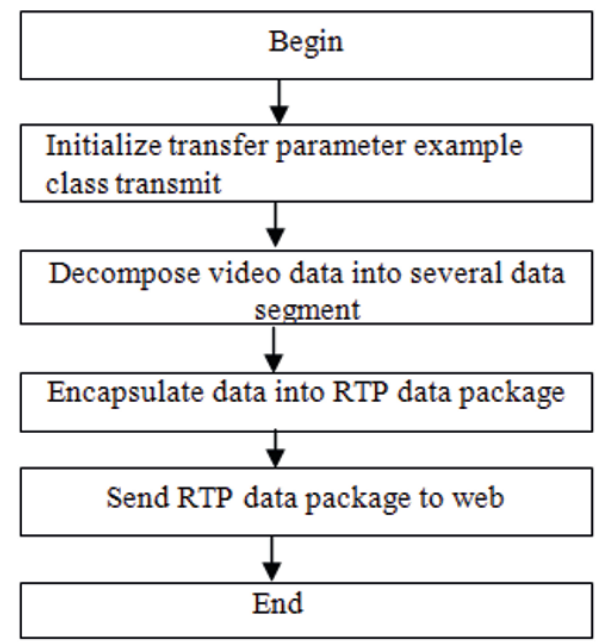

Fig.4: The flow image of sending video

The module of responding to users' requests responds to the instructions sent by users through the video server. The users send out a format instruction toward the particular port of the servers. After obtaining the instruction, according to analysis, the mold piece carries out a processing function to complete a concrete control. The module, in advance, keeps a form in which the orders and functions are match. After analyzing the order, 
then the performance function can be found in the form in response. With the development of the functions, the form can enlarge continuously, which can accomplish the modifying of the parameters for some servers and drive the camera to move and so on.

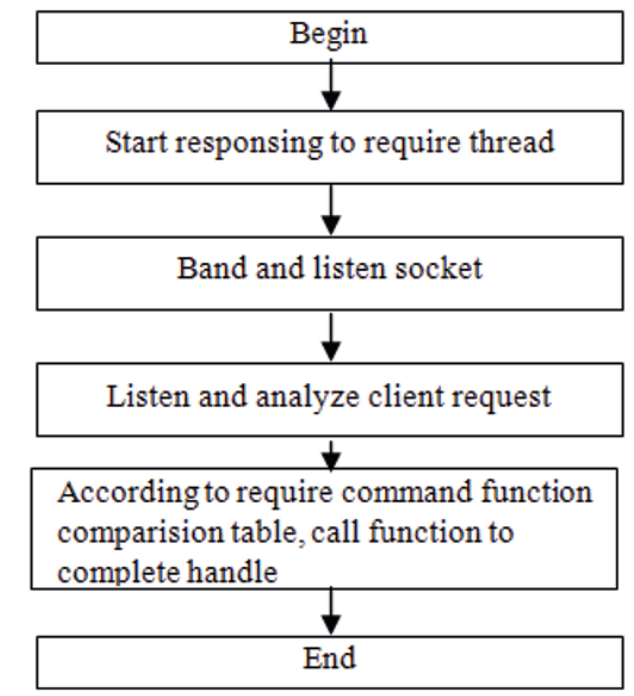

Fig.5: The flow image of answering the client's request

\section{EXPERIMENT}

The monitoring system was applied in the vegetable shed in Jiangsu University, with the net environment of a 10/100 M net, and hubs were used to connect users and the ports of video servers. Cameras are used to collect spot images. Users should have computers with Intel(R) Pentium(R) four CPU @ 2.66 GHz, 64M video card, 512M memory, 10/100 M gateway, 80G hard disk, and windows XP operating system for the Chinese version. When users carry an IE browser toward the servers to ask for live video streaming, servers can send out a video frequency flow at once. Without obvious wobble and delaying, users are able to get real-time information and clear pictures, which can be played fluently. When several users send out claims in the meantime toward the server, it can carry out multicast, and can satisfy the segmentation switch of daily pictures. It can also playback live video and signal scene. Especially, in the bad environment outside, it can also carry on the real-time monitoring normally. 


\section{CONCLUSION}

Aiming at the practical application needs of the video surveillance of the pump station, we design a set of build-in network video frequency supervision system with several new technologies such as coding image acquisition, build-in system and network technique. Based on the ARM7 chips of S3C44BOX and build-in Linux operating system, the system adopts cameras to catch the video frequency, and after compressing codes by MPEG-4 chip, it can connect the network directly. With standard network browser and the medium player procedure, users can observe the long range video frequency images inside the big shed. It has certain expansion meaning and can satisfy the need of the safe supervision management for the pump station at present.

\section{ACKNOWLEDGEMENTS}

Funding for this research was provided by China Postdoctoral Science Foundation under Grant (NO:20070420972); Jiangsu University High-grade Specialty Person Scientific Research Foundation under Grant(NO:05JDG050); Changzhou Young Scientific and Technological Talent Training Plan (NO:CQ2008009).Jiangsu University College Student Scientific Research Project(NO:06A110).

\section{REFERENCES}

Behzad, M. Fault diagnosis of a centrifugal pump by vibration analysis, Proceedings of the 7th Biennial Conference on Engineering Systems Design and Analysis, 2004,3:221-226

He Zhi-xia, YUAN Jian-ping, LI De-tao, ZHANG Li-qun. Performance Prediction for Pumping Station System Based on Neural Network Technique. Journal of Jiangsu University(Natural Science Edition), 2003,24(4):45-48(in Chinese)

Jia Guo-fang. Research of the online Intelligent Monitoring Technology of the Water Pump. Drainage and Irrigation Machinery.2005(6):28-30(in Chinese)

Kallesoe,Carsten Skovmose. Model based fault diagnosis in a centrifugal pump application using structural analysis. Proceedings of the IEEE International Conference on Control Applications, 2004, 2:1229-1235

Xue Yun-hui. Image inspect system in Jianbi pump station. Drainage and Irrigation Machinery. 2006(6):27-30(in Chinese)

Zhang Feng-chen, ZHANG Xiao-jing, FENG Qin. Remote Monitoring System on the Pump Station of TM1300. Journal of Heilongjiang Hydraulic Engineering College. 2006,33(2):90-92(in Chinese)

Zhang Haiying, $\mathrm{Hu}$ Bin, ZHAO Xingtian, WU Shengyan. Design of Analog Video Conversion Interface . Electronic Engineer,2003(01):53-54(in Chinese)

Zhang Shaobin, LIN Li. Design of Numerical Remote Concentration Surveillance System Based on MPEG-4. Computer Engineering. 2007,33(1):265 267(in Chinese) 